\title{
An Overview on Anisotropy and its Geological Constrains
}

\author{
Gustavo Teixeira*12, Luiz Alberto Santos ${ }^{12}$ e Marco Cetale ${ }^{2}$, ${ }^{1}$ Petrobras S/A, ${ }^{2}$ UFF/DOT/GISIS
}

Copyright 2021, SBGf - Sociedade Brasileira de Geofísica.

This paper was prepared for presentation at $17^{\circ}$ International Congress of the Brazilian Geophysical Society, held in Rio de Janeiro, Brazil, August 16-19, 2021. Contents of this paper were reviewed by the Technical Committee of the $17^{\circ}$ International Congress of the Brazilian Geophysical Society. Ideas and concepts of the text are authors' responsibility and do not necessarily represent any position of the SBGf, its officers or members. Electronic reproduction or storage of any part of this paper for commercial purposes without the written consent of The Brazilian Geophysical Society is prohibited.

\section{Abstract}

The academy and seismic industry have shown the importance of considering anisotropy in velocity model building. There are several examples concerning imaging problems whenever anisotropic effects are neglected. By means of travel time matrices and seismic modeling, we analyze the effects of the two anisotropic classes (intrinsic and extrinsic), seeking to evaluate them separately and together. The results shows that for intercalations between shales and sandstones with low velocity contrast, the extrinsic anisotropic is very weak, with clear predominance of the shale intrinsic anisotropy.

\section{Introduction}

A long time ago, velocity models used for seismic imaging processes considered the physical medium as isotropic, assuming this assumption due to the greater operational ease in the implementation of the algorithms, as well as understanding that such methodology was enough for an adequate seismic imaging.

Thomsen (1986) is a paramount paper that marks a starting point for considering anisotropy in seismic processing. In recent decades, papers such as Cetale Santos et al. (2004), Berryman (2008), Kumar (2013) and Nunes et al. (2019) have revived the discussion about the importance of considering anisotropy in velocity models. They have shown that reflector positioning and amplitude problems can be mitigated when the medium is treated according to its anisotropic characteristics.

According to Romanelli (2013), it is possible to distinguish two types of anisotropy: intrinsic and extrinsic. The first is related to the characteristic properties of a specific lithology, as for instance in the case of shales, where the minerals alignment can generates a prominent anisotropic effect (Figure 1a).

The extrinsic case, on the other hand, is the result of interleaving between different layers - where each thickness has subseismic character $(\mathrm{t})$ - composing a larger layer $(T)$, which can be identified in the seismic records (Figure 1b).

As indicated by Valcke et al. (2006), major factors for intrinsic anisotropy are: crystals preferential orientation, variations in the spatial distribution of minerals, morphology of grains, alignment of fractures and pores, beyond the type of pore filling material.

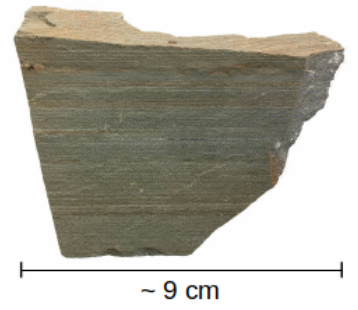

(a)

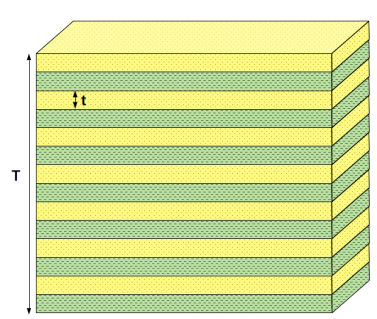

(b)
Figure 1: a) Laminated shale sample (Source: https://www.sandatlas.org/shale/), b) Interleaving model with shale and sandstone layers.

One of the most important papers within this theme is from Thomsen (1986), due to its formulations opened the perspective of more practical and relatively simpler applications for the geophysics applied to the exploration and production of hydrocarbons.

Thomsen op. cit. considers the premise that most cases of interest within the oil industry are composed by lithologies with relatively weak to moderate anisotropies, less than $20 \%$. He presents three parameters to describe the anisotropic characteristics of a rock, which are: Epsilon $(\varepsilon)$, Gamma $(\gamma)$ and Delta $(\delta)$.

Thomsen's parameters can then be understood as derivations of the stiffness matrix (Equation 1), which represents the physical properties of each material. This matrix is responsible for weighing the resulting deformations from the application of a given stress, treating the contribution of each of its elements individually, according to the Hooke's law:

$$
\begin{gathered}
\sigma_{i j}=C_{i j k l} e_{k l} \\
\mathrm{C}_{\mathrm{mn}}=\left[\begin{array}{llllll}
\mathrm{C}_{11} & \mathrm{C}_{12} & \mathrm{C}_{13} & \mathrm{C}_{14} & \mathrm{C}_{15} & \mathrm{C}_{16} \\
& \mathrm{C}_{22} & \mathrm{C}_{23} & \mathrm{C}_{24} & \mathrm{C}_{25} & \mathrm{C}_{26} \\
& & \mathrm{C}_{33} & \mathrm{C}_{34} & \mathrm{C}_{35} & \mathrm{C}_{36} \\
& & & \mathrm{C}_{44} & \mathrm{C}_{45} & \mathrm{C}_{46} \\
& & & & \mathrm{C}_{55} & \mathrm{C}_{56} \\
& & & & & \mathrm{C}_{66}
\end{array}\right]
\end{gathered}
$$

In a simplified way, three main forms of anisotropy arrangement can be indicated, with characteristic representations in the stiffness matrix, corresponding to different orientations in the symmetry axis. Such models 
can be combined, creating more complex anisotropic contexts.

At first, we have a Vertical Transversely Isotropic (VTI) medium, represented by the figure $1 \mathrm{~b}$, where the axis of symmetry is vertical, being this the model considered for the formulations of this work.

Additionally, it is possible to indicate the Horizontal Transversely Isotropic ( $\mathrm{HTI})$ and Tilted Transversely Isotropic (TTI) media. An HTI case has a horizontal axis of symmetry (in the example of the figure $1 \mathrm{~b}$, the layers would be in the vertical direction). The TTI medium considers the axis of symmetry inclined, with an orientation between the VTI and HTI cases.

According to Costa e Silva (1995) and Rosa-Filho (2002), Thomsen's formulations can be presented as follows, valid for VTI media:

$$
\begin{gathered}
\varepsilon \equiv \frac{C_{11}-C_{33}}{2 C_{33}}=\frac{V_{P}\left(90^{\circ}\right)-V_{P}\left(0^{\circ}\right)}{2 V_{P}\left(0^{\circ}\right)}, \\
\gamma \equiv \frac{C_{66}-C_{44}}{2 C_{44}} \\
\delta \equiv \frac{2\left(C_{13}+C_{44}\right)-\left(C_{33}-C_{44}\right)\left(C_{11}+C_{33}-2 C_{44}\right)}{2 C_{44}}=\frac{1}{2}\left(\frac{V_{I N T}^{2}}{V_{P}^{2}\left(0^{\circ}\right)}\right), \\
C_{33}=\rho V_{P V} \\
C_{55}=C_{44}=\rho V_{S V}
\end{gathered}
$$

Additionally, we can indicate the formulations for the other elements of the matrix as they are organized in Kumar (2013):

$$
\begin{gathered}
C_{11}=(1+2 \varepsilon) C_{33} \\
C_{66}=(1+2 \gamma) C_{44} \\
C_{13}=\sqrt{2 \delta C_{33}\left(C_{33}-C_{55}\right)+\left(C_{33}-C_{55}\right)^{2}}-C_{55}
\end{gathered}
$$

Singh and Sircar (2014) evaluated possible variations in shale anisotropy from measurements of velocity in samples subjected to increasing confining pressures, thus simulating different confining pressure conditions. The results obtained indicate that in the proposed experiment, there is initially a greater increase in the velocity propagation of the $\mathrm{P}$ wave in the horizontal direction (parallel to the bedding) than in the vertical direction. However, the continuity of the test also indicated that after a certain pressure, the vertical velocity starts to increase at a higher rate than the horizontal speed (Figure 2).

The behavior described for the shale when it is subjected to pressure increases then indicates that the original anisotropy of the rock shows a small growth in the initial

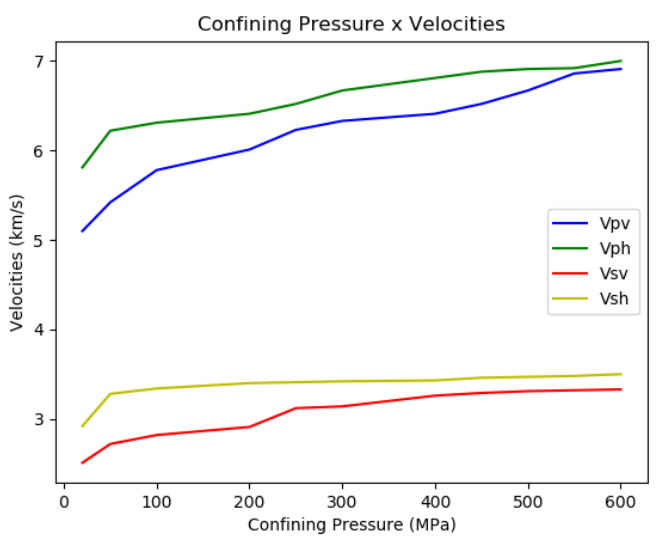

Figure 2: Velocity variation as a function of confining pressure (Modified from Singh and Sircar, 2014).

phase, subsequently decreasing, from the point where the propagation velocity in the vertical direction starts to increase more rapidly than the velocity in the horizontal direction (Figure 3).

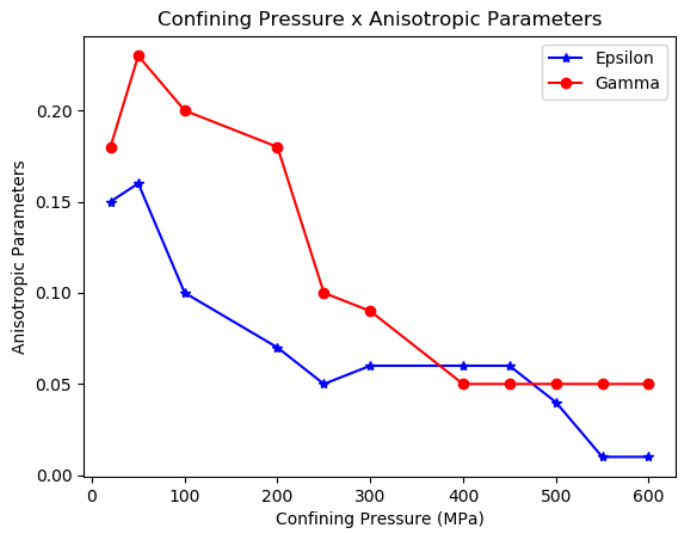

Figure 3: Anisotropic parameters variation as a function of confining pressure (Modified from Singh and Sircar, 2014).

Singh and Sircar (2014) suggest that such behavior is result from the microcracks, low aspect ratio pores and fractures closing. This process is specially evident from the pressure of $50 \mathrm{MPa}$, when there is a greater velocity increase in the vertical direction. Adicionally, the autors indicate a bigger change in the Young's modulus in the vertical direction, being too a result of the closing of microcracks and fractures parallels to the bedding.

\section{Materials and Methods}

Two experiments were carried out for this work, aiming to present the effects of anisotropy on wave propagation. The first one consists of estimative of transit time matrices (MTT), with results calculated for an isotropic medium and an anisotropic medium.

The second experiment consists of elastic modeling of wave propagation, seeking to demonstrate the differences in the wave fields obtained from the isotropic and anisotropic media, thus allowing the comparison between 
them.

The analytical estimate of velocities as a function of the propagation angle and seismic modeling were elaborated from the data available in Sondergeld and Rai (2011) for shale and sandstone (Table 1).

Table 1: Rocks parameters.

\begin{tabular}{|c|c|c|c|c|c|c|}
\hline & $\begin{array}{c}\rho \\
(\mathrm{gm} / \mathrm{cc})\end{array}$ & $\begin{array}{c}\mathrm{Vp} \\
(\mathrm{m} / \mathrm{s})\end{array}$ & $\begin{array}{c}\mathrm{Vs} \\
(\mathrm{m} / \mathrm{s})\end{array}$ & $\varepsilon$ & $\gamma$ & $\delta$ \\
\hline Shale & 2.42 & 3060 & 1490 & 0.256 & 0.481 & -0.051 \\
Sand & 2.0 & 2950 & 1480 & 0 & 0 & 0 \\
\hline
\end{tabular}

\section{Travel Time Matrices}

For the initial presentation of the impacts of anisotropy, transit time matrices were generated from the formulations of Thomsen (1986) and Berryman (2008). These matrices allow a preliminary analysis to the modeling stage, indicating how the wave propagation in the referred medium should occur.

The algorithm built for the MTT calculation has as reference the central point of a grid, from which, for each of the cells, the distance and angle between them and the referred point is calculated. Based on this information, it is possible to calculate the velocity to be applied and subsequently estimate the transit time.

Thomsen's (1986) equations used to calculate velocities as a function of the propagation angle aim to represent media with relatively weak anisotropy (Equations 10 and 11), while Berryman (2008) broadens the scope to contexts of moderate and strong anisotropy (Equations 12, 13, 14 and 15).

$$
\begin{gathered}
V_{p}(\theta) \approx \alpha_{0}\left(1+\delta \sin ^{2} \theta \cos ^{2} \theta+\varepsilon \sin ^{4} \theta\right), \\
V_{S V}(\theta) \approx \beta_{0}\left[1+\frac{\alpha_{0}^{2}}{\beta_{0}^{2}}(\varepsilon-\delta) \sin ^{2} \theta \cos ^{2} \theta\right],
\end{gathered}
$$

where: $V_{p}(\theta)$ - P velocity by angle, $V_{S V}(\theta)$ - S velocity by angle, $\alpha_{0}$ - P velocity in vertical direction and $\beta_{0}-\mathrm{S}$ velocity in vertical direction.

$$
V_{p}(\theta) / V_{p}(0) \approx 1+\varepsilon \sin ^{2} \theta-(\varepsilon-\delta) \frac{2 \sin ^{2} \theta_{m} \sin ^{2} \theta \cos ^{2} \theta}{1-\cos 2 \theta_{m} \cos 2 \theta},
$$

$V_{s v}(\theta) / V_{s}(0) \approx 1+\left(V_{p}^{2}(0) / V_{s}^{2}(0)\right)(\varepsilon-\delta) \frac{2 \sin ^{2} \theta_{m} \sin ^{2} \theta \cos ^{2} \theta}{1-\cos 2 \theta_{m} \cos 2 \theta}$.

where: $V_{p}(0)$ - P velocity in vertical direction, $V_{s}(0)-\mathrm{S}$ velocity in vertical direction and:

$$
\begin{gathered}
\sin ^{2} \theta_{m}=\frac{V_{p}^{2}(0)-V_{s}^{2}(0)}{2\left[(1+\varepsilon) V_{p}^{2}(0)-V_{s}^{2}(0)\right]}, \\
\cos 2 \theta_{m}=\frac{\varepsilon V_{p}^{2}(0)}{(1+\varepsilon) V_{p}^{2}(0)-V_{s}^{2}(0)}
\end{gathered}
$$

\section{Seismic Modeling}

The algorithm implemented for modeling is based on Martins (2003), built from the staggered finite difference grid of Levander (1988). An important feature of this methodology is its versatility, being efficiently applied in contexts of low and high anisotropy values. The general formulations used are the following, valid for VTI medium:

$$
\begin{gathered}
\rho \frac{\delta U}{\delta t}=\frac{\delta \tau_{x x}}{\delta x}+\frac{\delta \tau_{x z}}{\delta z} \\
\rho \frac{\delta V}{\delta t}=\frac{\delta \tau_{z x}}{\delta x}+\frac{\delta \tau_{z z}}{\delta z} \\
\frac{\delta \tau_{x x}}{\delta t}=C_{11} \frac{\delta U}{\delta x}+C_{13} \frac{\delta V}{\delta z} \\
\frac{\delta \tau_{z z}}{\delta t}=C_{33} \frac{\delta V}{\delta z}+C_{13} \frac{\delta U}{\delta x} \\
\frac{\delta \tau_{z x}}{\delta t}=C_{44}\left(\frac{\delta U}{\delta z}+\frac{\delta V}{\delta x}\right)
\end{gathered}
$$

For these equations, $\mathrm{U}$ and $\mathrm{V}$ are the horizontal and vertical components of the particle velocity field; $\tau_{x x}, \tau_{z z}$ e $\tau_{z x}$ are the stresses; and $C_{m n}$ corresponds to elastic parameters.

Three simulations is carried out in this stage: the first considers a homogeneous isotropic medium, thus serving as a reference for the other models. The values of $\mathrm{Vp}, \mathrm{Vs}$ and density are calculated from the harmonic mean of the original shale and sandstone parameters.

In the second simulation, the seismic modeling is performed in the context of intercalation between layers of shale and sandstone, assuming shale as isotropic (disregarding the parameters $\varepsilon$ and $\delta$ ). We consider the proportion of $50 \%$ for each lithology, distributed interleaved and evenly.

Finally, a simulation of intercalation between shales and sandstones is made, this time considering the intrinsic shale anisotropy (using the parameters $\varepsilon$ and $\delta$ ). Similary to the previous case, we consider equal proportions for both lithologies.

For all simulations, the dominant frequency of $40 \mathrm{~Hz}$ and sample rate of 0.00025 seconds are used. For interbed layers cases, the thickness of each one is 5 meters.

\section{Results}

The first strategy for visualization and analysis of velocities as a function of the propagation angle of a seismic wave can be seen in the figures 4 and 5 , where the values of $\mathrm{Vp}$ and Vsv are plotted.

It is possible to indicate that for the two formulations used (Thomsen and Berryman), the calculated primary wave velocity for $90^{\circ}$ angle has the same value for both. Differences in velocity values are observed especially for angles in the range between $20^{\circ \circ}$ and $80^{\circ}$.

Taking as an example the angle of $60^{\circ}$ and the velocity of the compressional wave, we have the estimated value 


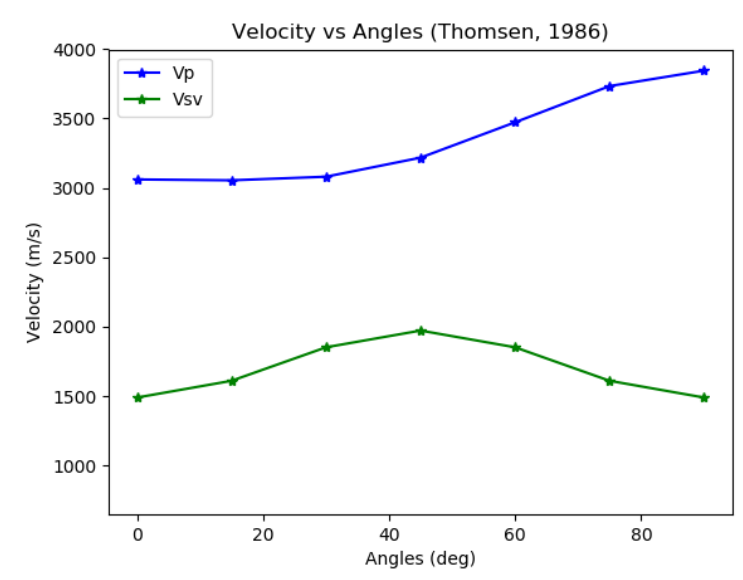

Figure 4: Velocities as function of propagation angle using Thomsen's equations (1986).

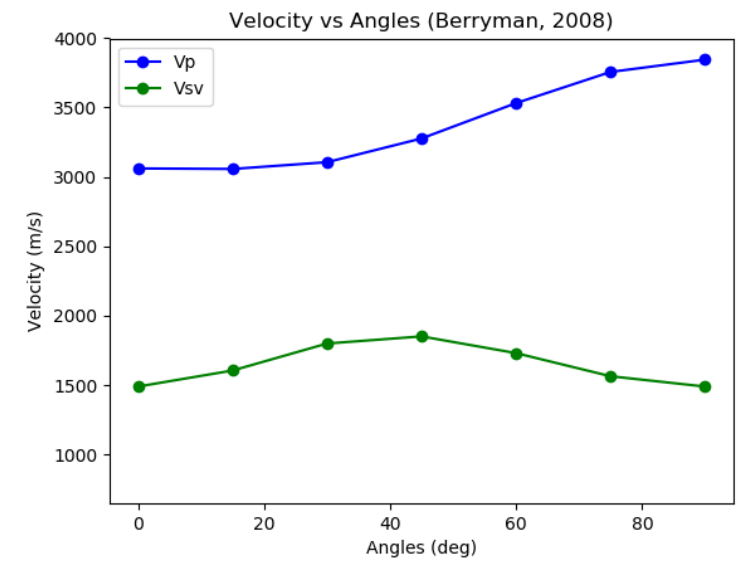

Figure 5: Velocities as function of propagation angle using Berryman's equations (2008).

at $3471 \mathrm{~m} / \mathrm{s}$ for Thomsen and $3530 \mathrm{~m} / \mathrm{s}$ for Berryman (Figures 4 and 5). This difference causes a significant discrepancy between the travel time matrices elaborated from the different equations.

Then having the velocities available as function of the propagation angle, it is possible to calculate the corresponding travel time matrices (MTT). For this experiment, wave propagation was considered only in the shale.

In the figure 6 we have the MTT presentation for three situations: a) isotropic medium (using only the shale $\mathrm{Vp}$ ); b) anisotropic medium, using the equations of Thomsen (1986) and; c) anisotropic medium, using Berryman's equations (2008), both using all elastic parameters of the shale.

Thus, considering the parameters used in these simulations, we have a more elliptical morphology in the MTT representation calculated from Berryman (2008), while in the MTT calculated from Thomsen (1986) we have a more lozenge-shape feature.
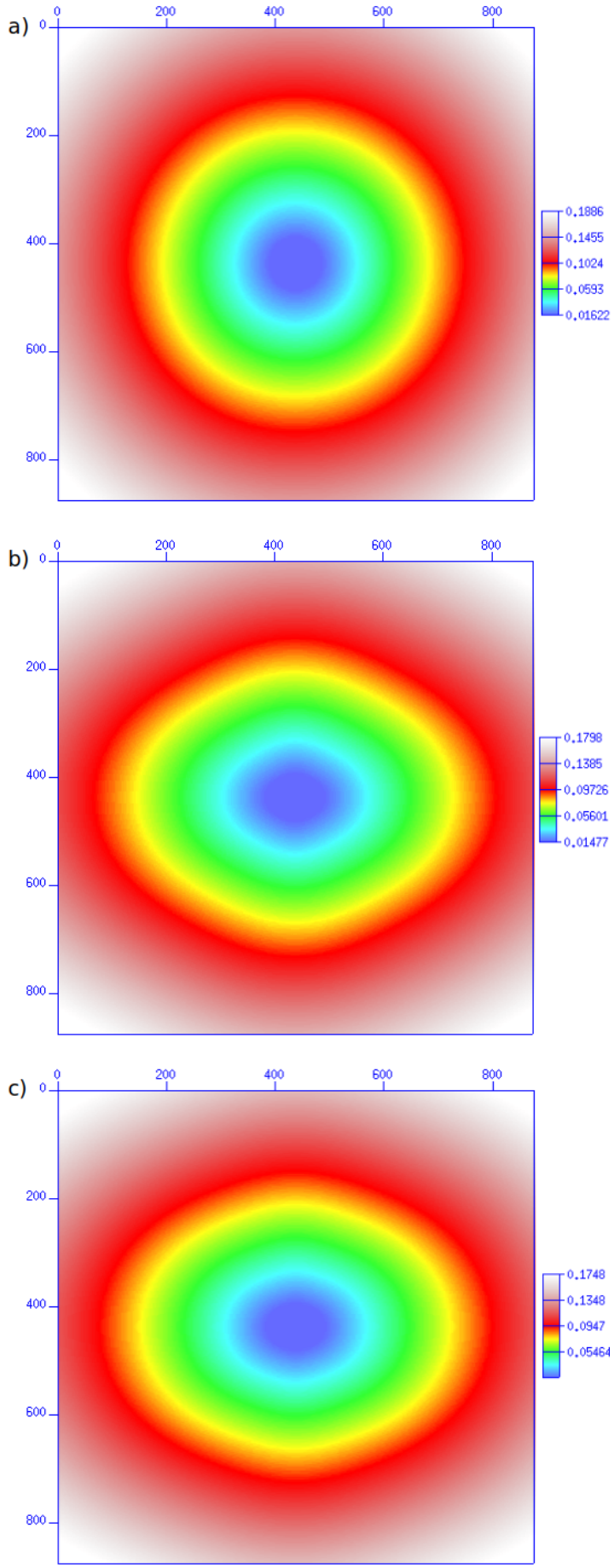

Figure 6: Travel time matrices: a) isotropic medium; b) anisotropic medium - Thomsen's formulations (1986); b) anisotropic medium - Berryman's formulations (2008);

The presentation of the effects of intrinsic and extrinsic anisotropy is done in the second experiment (7), where we have the results of wave propagation models in different contexts.

At first, we have an example of propagation in a homogeneous isotropic medium, resulting in a spherical 
feature, where there is just the propagation of a $\mathrm{P}$ wave.

The second seismic modeling indicates that the anisotropic effect resulting from the model with intercalations is practically imperceptible, suggesting that the velocity difference between the layers is not enough to generate a significant extrinsic anisotropy. It is possible to observe the occurrence of internal features of low amplitude, formed due to the various interleaved layers.

In the last modeling we consider the two classes of anisotropy (intrinsic and extrinsic). In this experiment the intrinsic shale anisotropy has a dominant character, contributing to the generation of a prominent $S$ wave, not visible in the past example. The shape of the wave field approximates the features observed in the travel time matrices, confirming the analytical predictions.

\section{Discussion and Conclusion}

The bibliographic review presented in this work, as well as the results displayed, contribute to the understanding of the origin and effects resulting from anisotropy, especially in seismic waves propagation.

The travel time matrices (MTT) generated allow to visualize the differences between the formulations of Thomsen (1986) and Berryman (2008) for the proposed case, showing how they work differently for the intermediate angles.

The presented wave fields suggest the dominance of intrinsic anisotropy over extrinsic, indicating that the velocity contrast between layers present in an interleaving must be high for a significant extrinsic anisotropic effect to occur.

\section{Acknowledgments}

The authors would like to thank Petrobras S/A and the Seismic Inversion and Imaging Group (GISIS-UFF) for their support for development of this work.

\section{References}

Berryman, J. G., jan 2008, Exact seismic velocities for transversely isotropic media and extended Thomsen formulas for stronger anisotropies: GEOPHYSICS, 73, no. 1, D1-D10.

Cetale Santos, M. A., Soares Filho, D. M., and Osório, P. L. M., jan 2004, A finite difference scheme for locally transverse isotropic media applied to a highly tectonic deformed model: A finite difference scheme for locally transverse isotropic media applied to a highly tectonic deformed model:, Society of Exploration Geophysicists, SEG Technical Program Expanded Abstracts 2004, 1913-1916.

Costa e Silva, M. B., 1995, Influência da anisotropia VTI na correção de sobretempo normal em dados sísmicos e análise de velocidade por gradiente descendente: Ph.D. thesis, PUC-Rio.

Kumar, D., oct 2013, Applying Backus averaging for deriving seismic anisotropy of a long-wavelength equivalent medium from well-log data: Journal of Geophysics and Engineering, 10, no. 5, 055001.

Levander, A. R., nov 1988, Fourth-order finite-difference
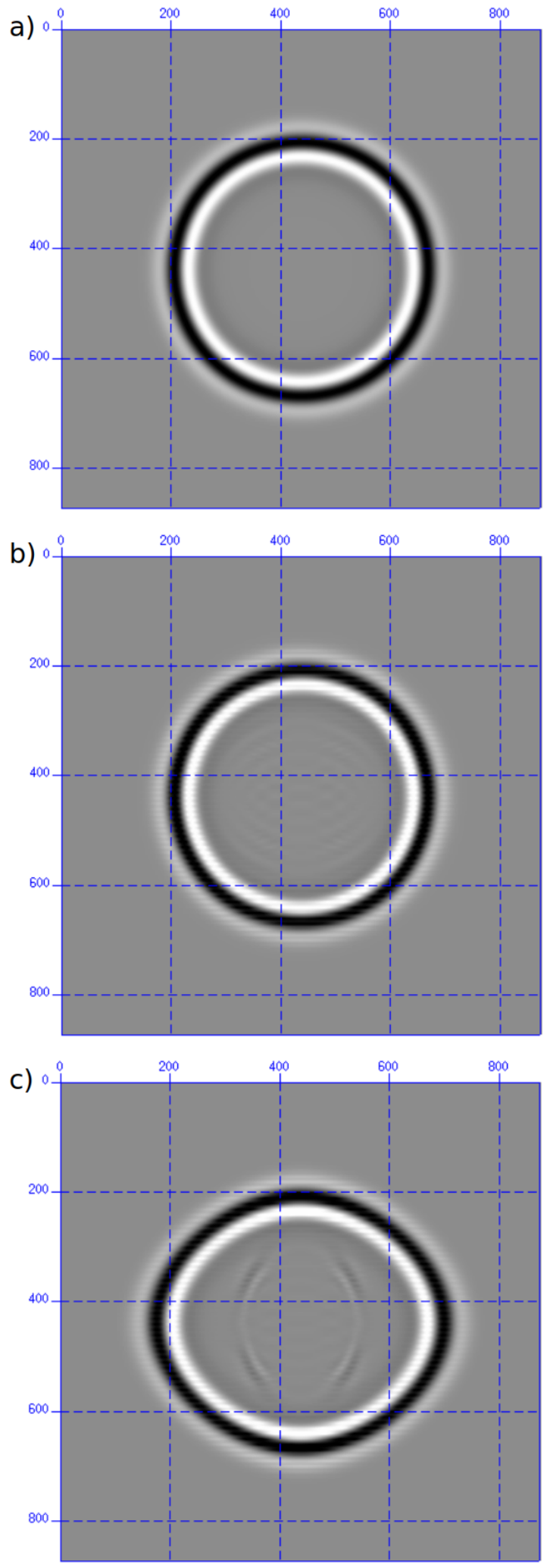

Figure 7: Pressure fields for wave propagation in the media: a) isotropic, b) only extrinsic anisotropy, c) intrinsic and extrinsic anisotropies. 
P-SV seismograms: GEOPHYSICS, 53, no. 11, 14251436.

Martins, E. O., 2003, Modelagem Sísmica em Meios Complexos: Ph.D. thesis, Federal University of Rio de Janeiro.

Nunes, C., Mesz, L., de Carvalho, A., and Carneiro, V., 2019, Investigation of anisotropy as a possible cause for an AVO pitfall - post-mortem analysis: Investigation of anisotropy as a possible cause for an AVO pitfall post-mortem analysis:, Brazilian Geophysical Society, Proceedings of the 16th International Congress of the Brazilian Geophysical SocietyExpogef, 1-5.

Rosa-Filho, J. C., 2002, Modelagem Sísmica de Ondas Elásticas e Migração Reversa no tempo em Meios Transversalmente Isotrópicos: Ph.D. thesis, Federal University of Rio de Janeiro.

Singh, A., and Sircar, A., 2014, Sources and Measurement of Velocity Anisotropy of Cambay Shale, Cambay Basin, India: IJLTEMAS, pages 169-179.

Sondergeld, C. H., and Rai, C. S., mar 2011, Elastic anisotropy of shales: The Leading Edge, 30, no. 3, 324331.

Thomsen, L., oct 1986, Weak elastic anisotropy: GEOPHYSICS, 51, no. 10, 1954-1966.

Valcke, S. L. A., Casey, M., Lloyd, G. E., Kendall, J.-M., and Fisher, Q. J., aug 2006, Lattice preferred orientation and seismic anisotropy in sedimentary rocks: Geophysical Journal International, 166, no. 2, 652-666. 